\title{
Construction and analysis of circular RNA molecular regulatory networks in clear cell renal cell carcinoma
}

\author{
CHUANYU MA，JIE QIN，JUNPENG ZHANG，XINGLI WANG，DONGJUN WU and XIUNAN LI
}

\begin{abstract}
Department of Urology, The First Affiliated Hospital of Dalian Medical University, Dalian, Liaoning 116600, P.R. China
\end{abstract}
Received March 31, 2019; Accepted October 15, 2019

DOI: $10.3892 / \mathrm{mmr} .2019 .10811$

\begin{abstract}
Increasing evidence has indicated that circular (circ)RNAs participate in carcinogenesis; however, the specific regulatory mechanisms underlying the effects of circRNAs, microRNAs (miRNAs/miRs) and genes on the development of clear cell renal cell carcinoma (CCRCC) remain unclear. In the present study, RNA microarray data from CCRCC tissues and control samples were downloaded from the Gene Expression Omnibus and The Cancer Genome Atlas, in order to identify significantly dysregulated circRNAs, miRNAs and genes. The Cancer-Specific circRNA Database was used to explore the interactions between miRNAs and circRNAs, whereas TargetScan and miRDB were employed to predict the mRNA targets of miRNAs. Functional enrichment and prognostic analyses were conducted in $\mathrm{R}$. The results revealed that 324 circRNAs were downregulated, whereas 218 circRNAs were upregulated in cancer. In addition, a circRNA-miRNA-mRNA interaction network was constructed. Gene Ontology analysis of the upregulated genes revealed that these genes were enriched in biological processes, including 'flavonoid metabolic process', 'cellular glucuronidation' and 'T cell activation'. The downregulated genes were mainly enriched in biological processes, such as 'nephron development', 'kidney development' and 'renal system development'. The hub genes, including membrane palmitoylated protein 7, aldehyde dehydrogenase 6 family member A1, transcription factor AP- $2 \alpha$, collagen type IV $\alpha 4$ chain, nuclear receptor subfamily 3 group $\mathrm{C}$ member 2 , plasminogen, Holliday junction recognition protein, claudin 10 , kinesin family member $18 \mathrm{~B}$ and thyroid hormone receptor $\beta$, and the hub miRNAs, including miR-21-3p, miR-155-3p, miR-144-3p, miR-142-5p, miR-875-3p, miR-885-3p, miR-3941, miR-224-3p, miR-584-3p and miR-138-1-3p, were significantly associated with CCRCC survival. In conclusion, these results suggested that the significantly dysregulated circRNAs, miRNAs and genes identified
\end{abstract}

Correspondence to: Dr Xiunan Li, Department of Urology, The First Affiliated Hospital of Dalian Medical University, 5 Longbin Road, Dalian, Liaoning 116600, P.R. China

E-mail: wawama_@126.com

Key words: circular RNA, microRNA, clear cell renal cell carcinoma, prognosis in this study may be considered potential biomarkers of the carcinogenesis of CCRCC and the survival of patients with this disease.

\section{Introduction}

Renal cell carcinoma (RCC) is a common type of cancer that is derived from renal tubular epithelial cells (1). Clear cell RCC (CCRCC) has been reported to be the most common histological subtype of $\operatorname{RCC}(2,3)$. As for clinical treatment, RCC is often resistant to radiotherapy, chemotherapy and hormonal treatments (4). Although surgical resection can effectively treat CCRCC, $20-40 \%$ of patients develop local recurrence or distant metastasis following surgery (5). The observed survival rate of advanced CCRCC is very low, which poses an obstacle in treating and managing patients with CCRCC (6). As CCRCC is a highly aggressive cancer with concomitant poor prognosis, reliable biomarkers for predicting the susceptibility and survival of patients with CCRCC are urgently required.

Circular RNAs (circRNAs) represent a series of endogenous RNAs that modulate the expression of genes and do not encode proteins (7). circRNAs are commonly characterized by their stabilized structure and tissue specificity, and are widely expressed in a variety of eukaryotic cells $(8,9)$. CircRNAs also have tissue specificity and their expression is tissue specific in the eukaryotic transcriptome (10). An investigation into the regulation of competing endogenous RNAs (ceRNAs) by Meng et al (9) provided insight into the complex post-transcriptional interaction network of various circRNAs and long non-coding RNAs; these molecules function as microRNA (miRNA/miR) sponges, suppressing their effects via miRNA response elements. Emerging evidence has suggested that circRNAs may be considered robust biomarkers and potential therapeutic targets in several diseases, including cancer (11).

Numerous studies have confirmed the existence of the regulatory role of ceRNAs in the circRNA-miRNA-mRNA network within various diseases, including renal cancer $(12,13)$. For example, the novel circRNA circHIAT1 has been reported to be downregulated in CCRCC tissues compared with normal tissues. Analysis of androgen receptor-inhibited circHIAT1 revealed the aberrant expression of miR-195-5p/29a-3p/29c-3p, which induced cell division cycle 42 expression, promoting the migration and invasion of CCRCC cells (12). Furthermore, a previous study demonstrated that knockdown of circRNA_0001451 significantly enhanced tumor proliferation in vitro; the levels 


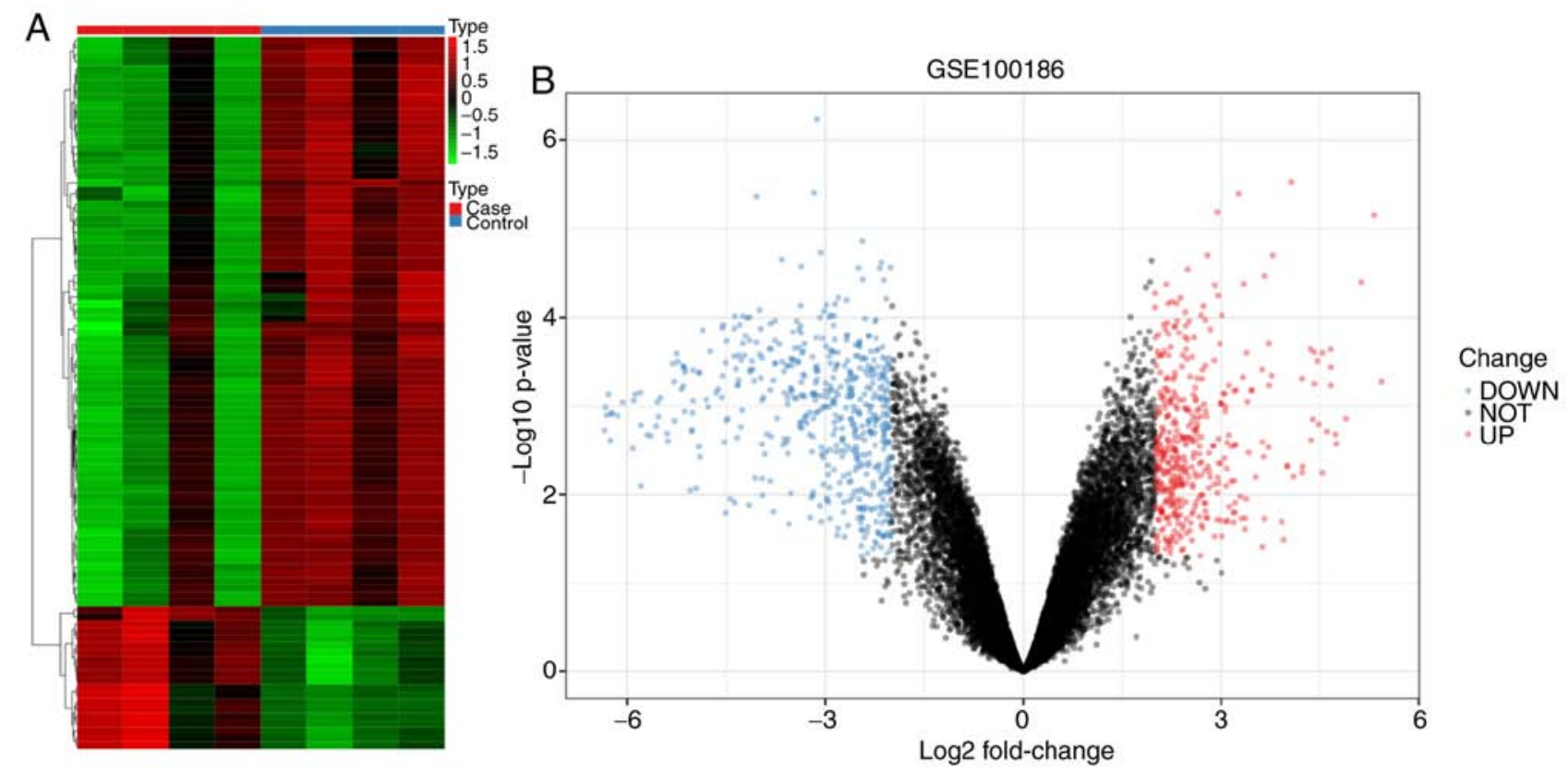

Figure 1. Differential expression analysis of microarray GSE100186. (A) Heatmap of GSE100186, in the heatmap, the green color represents low expression while the red color represents high expression. (B) Volcano plot of GSE100186. Compared with the normal group, red represents upregulated genes in the cancer group, whereas blue represents downregulated genes in the cancer group. NOT represents no change in the differential expression analysis.

of circRNA_0001451 were associated with the differentiation grade of patients with CCRCC (13). In addition, circRNA ZNF609 has been reported to serve as a ceRNA in modulating the expression of Forkhead box P4 via sponging miR-138-5p in renal cancer; high circ-ZNF609 expression was determined to enhance the growth and invasive characteristics of renal cancer cells (14).

Despite improved understanding of the association between circRNA expression and various types of human cancer, the role of circRNAs in renal cancer remains unclear. The present study identified several differentially expressed circRNAs, miRNAs and genes by analyzing datasets of the Gene Expression Omnibus (GEO, https://www.ncbi.nlm.nih.gov/gds/) and The Cancer Genome Atlas (TCGA, https://www.cancer.gov/about-nci/organization/ccg/research/structural-genomics/tcga) for CCRCC. Additionally, a circRNA-miRNA-mRNA regulatory network was constructed using bioinformatics tools. The present findings may improve understanding of the mechanisms underlying the carcinogenesis of CCRCC.

\section{Materials and methods}

Microarray database. To identify datasets, 'renal cellular cell carcinoma' and 'circRNA' were used as keywords to search the GEO; datasets including cancer and normal groups was the main inclusion criterion. The data were downloaded from the GEO of the National Center for Biotechnology Information repository. The GSE100186 (https://www.ncbi.nlm.nih. gov/geo/query/acc.cgi?acc=GSE100186) circRNA expression microarray dataset of CCRCC was used, which contained data from four CCRCC samples and four normal samples. Arraystar circRNA microarray (https://www.ncbi.nlm.nih. gov/geo/query/acc.cgi?acc=GPL21825) analysis was used to examine the expression of circRNAs in CCRCC and matched non-tumor tissues. mRNA expression and miRNA profiling of TCGA CCRCC data was performed to identify differentially expressed genes (DEGs) and differentially expressed miRNAs (DEMs) between cancer and normal tissues. TCGA data were downloaded from UCSC XENA (https://xena.ucsc.edu/).

Data processing. The limma package in $\mathrm{R}$ (version 3.6.0, https://www.r-project.org/) was used to analyze differentially expressed circRNAs (DECs) between the groups. The 'edge R' package (version 3.26.8; https://www.bioconductor.org/packages/release/bioc/html/edgeR.html) was employed to analyze the DEGs and DEMs between the groups. P $<0.05$ and llog fold changel $>2$ were applied to determine statistical significance.

circRNA-miRNA-mRNA regulatory network. The CancerSpecific circRNA Database (CSCD; http://gb.whu.edu.cn/ CSCD) can be used to predict interactions between circRNAs and miRNAs (15). Using the CSCD, miRNAs that interact with DECs were predicted. Subsequently, the DECs that interact with the miRNAs were identified as CCRCC-specific miRNAs. TargetScan 7.2 (http://www.targetscan.org/vert_72/) and miRDB 2.0 (http://www.mirdb.org/) were used to predict the target genes of DEMs, which were matched to genes with a mRNA expression profile that opposed the miRNA profile; the expression of miRNAs is often inversely associated with that of the target mRNA. Subsequently, the top five circRNAs with the highest degrees of connectivity were selected as the hub circRNAs. The circRNA-miRNA-mRNA regulatory network was constructed using Cytoscape 3.7.0 (https://cytoscape.org/).

Gene Ontology (GO) and pathway enrichment analyses. GO analysis is a useful bioinformatics strategy for annotating genes or gene products, and comprises three categories: Cellular component (CC), molecular function (MF) and biological 
process (BP) (16). Kyoto Encyclopedia of Genes and Genomes (KEGG) is a collection of databases, which contain comprehensive information regarding genomes, biological pathways, diseases and chemical substances (17). In the present study, GO and KEGG enrichment analyses were conducted using $\mathrm{R}$ package clusterprofiler (version 3.12.0; https://bioconductor. org/packages/release/bioc/html/clusterProfiler.html). $\mathrm{P}<0.05$ was considered to indicate a statistically significant difference.

Prognostic value of circRNA-regulated DEGs and DEMs. TCGA contains the survival information of patients with various types of cancer. Using the survival 2.44 package (https://cran.r-project.org/web/packages/survival/index.html) in $\mathrm{R}$, the prognostic value of circRNA-regulated DEGs and DEMs was assessed. Additionally, a survival curve was plotted using survminer 0.4.6 (https://cran.r-project.org/web/packages/survminer/index.html) package in R.

\section{Results}

Identification of DECs. Following analysis of differential expression, a total of 324 circRNAs in the GSE100186 dataset were downregulated in the cancer group, whereas 218 circRNAs were upregulated in the cancer group (Fig. 1 and Table SI). The top ten most significant circRNAs according to P-value included hsa_circ_0031594, hsa_circ_0001968, hsa_circ_0003596, hsa_circ_0058794, hsa_circ_0001873, hsa_circ_0003748, hsa_circ_0003997,hsa_circ_0000223, hsa_circ_0092367 and hsa_circ_0092360.

circRNA-miRNA-mRNA regulatory network. According to the CSCD datasets, 2,363 miRNAs were reported to interact with the identified DECs. After identifying the DECs that interact with the 2,363 miRNAs, 42 miRNAs were selected as CCRCC-specific miRNAs, including 32 upregulated miRNAs and 10 downregulated miRNAs. Using TargetScan, miRDB and TCGA-DEGs, a total of 244 downregulated genes and 85 upregulated genes were selected as circRNA-regulated genes. Subsequently, the circRNAs were sorted based on the degree of connectivity; the top five circRNAs were selected as hub circRNAs in the regulatory network of DEGs (Table I).

Construction of the circRNA-miRNA-upregulated mRNA network. As presented in Fig. 2A, a hub circRNA-miRNAupregulated mRNA network was built. The results of enrichment analysis of the 85 upregulated genes are presented in Fig. 2B and Table II. The upregulated genes were mainly enriched in BP, including 'flavonoid metabolic process', 'cellular glucuronidation' and 'T cell activation'. In addition, these DEGs were enriched in MF, including 'glucuronosyltransferase activity', 'UDP-glycosyltransferase activity' and 'protein heterodimerization activity'. KEGG pathway analysis suggested that DEGs were associated with 'ascorbate and aldarate metabolism', 'pentose and glucuronate interconversions', 'steroid hormone biosynthesis' and 'retinol metabolism'.

Construction of the circRNA-miRNA-downregulated $m R N A$ interaction network. As shown in Fig. 3A, a hub circRNA-miRNA-downregulated mRNA network was built.

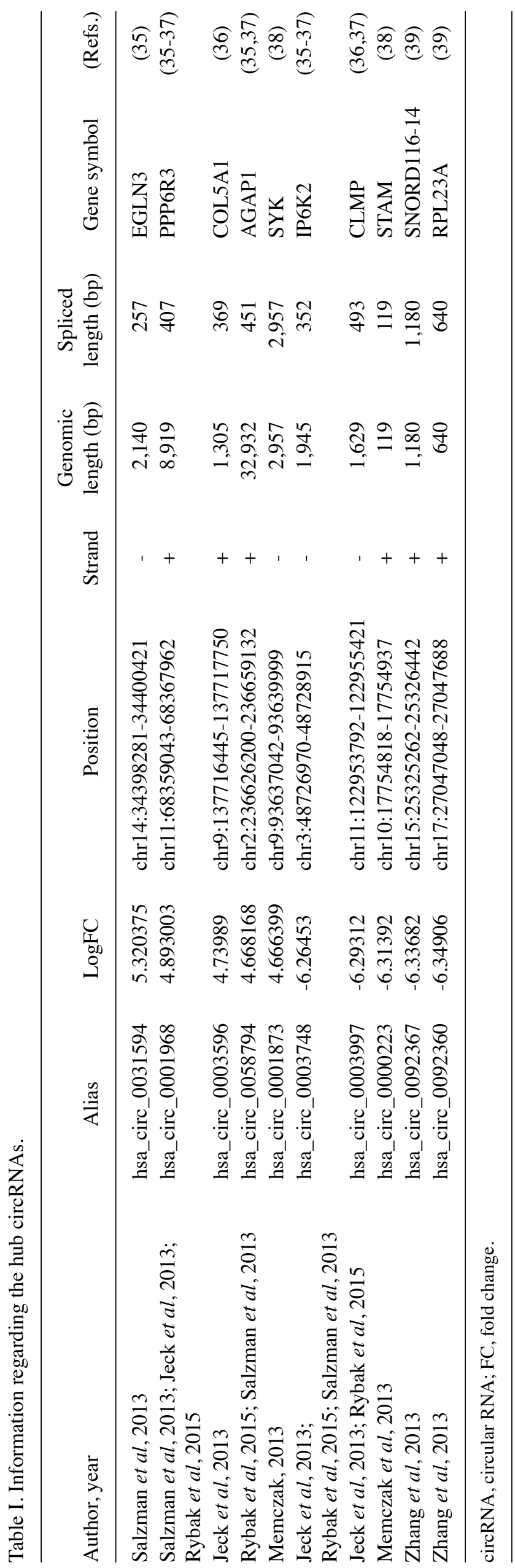


Table II. Top five GO terms and KEGG pathways enriched in the circRNA-miRNA-upregulated mRNA network.

\begin{tabular}{|c|c|c|c|c|c|}
\hline Term/pathway & ID & Description & Gene ratio & P-value & Count \\
\hline $\mathrm{BP}$ & GO:0009812 & Flavonoid metabolic process & $5 / 81$ & $5.62 \times 10^{-9}$ & 5 \\
\hline $\mathrm{BP}$ & GO:0052695 & Cellular glucuronidation & $5 / 81$ & $1.15 \times 10^{-8}$ & 5 \\
\hline BP & GO:0006063 & Uronic acid metabolic process & $5 / 81$ & $4.80 \times 10^{-8}$ & 5 \\
\hline $\mathrm{BP}$ & GO:0019585 & Glucuronate metabolic process & $5 / 81$ & $4.80 \times 10^{-8}$ & 5 \\
\hline $\mathrm{BP}$ & GO:0009410 & Response to xenobiotic stimulus & $7 / 81$ & $1.20 \times 10^{-6}$ & 7 \\
\hline MF & GO:0015020 & Glucuronosyltransferase activity & $5 / 78$ & $4.05 \times 10^{-7}$ & 5 \\
\hline MF & GO:0046982 & Protein heterodimerization activity & $10 / 78$ & $7.20 \times 10^{-5}$ & 10 \\
\hline MF & GO:0001228 & $\begin{array}{l}\text { DNA-binding transcription activator activity, } \\
\text { RNA polymerase II-specific }\end{array}$ & $9 / 78$ & $9.24 \times 10^{-5}$ & 9 \\
\hline MF & GO:0008194 & UDP-glycosyltransferase activity & $5 / 78$ & 0.000492 & 5 \\
\hline MF & GO:0005172 & $\begin{array}{l}\text { Vascular endothelial growth factor receptor } \\
\text { binding }\end{array}$ & $2 / 78$ & 0.001068 & 2 \\
\hline KEGG & hsa00053 & Ascorbate and aldarate metabolism & $5 / 42$ & $3.24 \times 10^{-7}$ & 5 \\
\hline KEGG & hsa00040 & Pentose and glucuronate interconversions & $5 / 42$ & $1.09 \times 10^{-6}$ & 5 \\
\hline KEGG & hsa00860 & Porphyrin and chlorophyll metabolism & $5 / 42$ & $3.21 \times 10^{-6}$ & 5 \\
\hline KEGG & hsa00140 & Steroid hormone biosynthesis & $5 / 42$ & $1.91 \times 10^{-5}$ & 5 \\
\hline KEGG & hsa00830 & Retinol metabolism & $5 / 42$ & $3.29 \times 10^{-5}$ & 5 \\
\hline
\end{tabular}

BP, biological process; GO, Gene Ontology; KEGG, Kyoto Encyclopedia of Genes and Genomes; MF, molecular function.

A

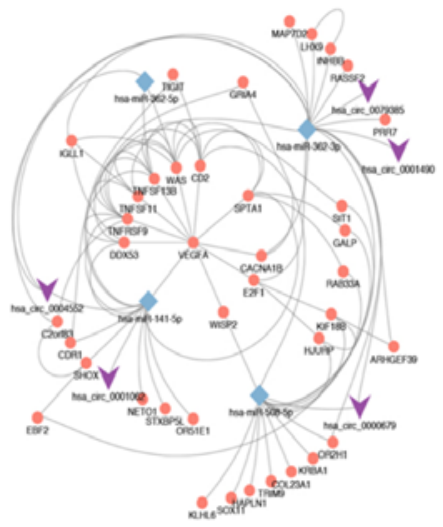

B
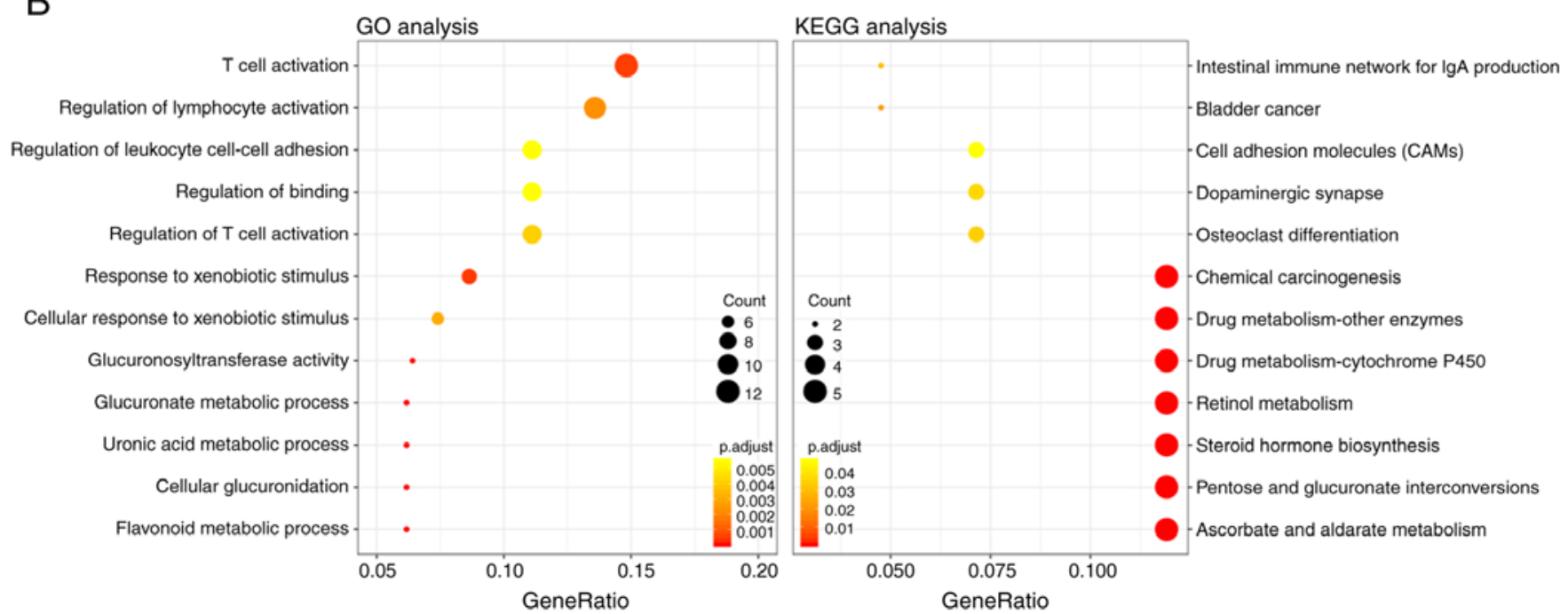

Figure 2. Enrichment analysis of the circRNA-miRNA-up-regulated mRNA network. (A) Regulatory network of hub circRNAs. Arrowheads represent circRNAs, diamonds represent miRNAs and circles represent genes. (B) Dotplot of GO and KEGG enrichment analyses. circRNA, circular RNA; GO, Gene Ontology; KEGG, Kyoto Encyclopedia of Genes and Genomes; miRNA, microRNA. 
Table III. Top five GO terms and KEGG pathways enriched in the circRNA-miRNA-downregulated mRNA network.

\begin{tabular}{|c|c|c|c|c|c|}
\hline Term/pathway & ID & Description & Gene ratio & P-value & Count \\
\hline BP & GO:0072006 & Nephron development & $14 / 230$ & $4.20 \times 10^{-9}$ & 14 \\
\hline BP & GO:0001822 & Kidney development & $18 / 230$ & $1.50 \times 10^{-8}$ & 18 \\
\hline $\mathrm{BP}$ & GO:0072001 & Renal system development & $18 / 230$ & $3.72 \times 10^{-8}$ & 18 \\
\hline BP & GO:0072073 & Kidney epithelium development & $13 / 230$ & $4.06 \times 10^{-8}$ & 13 \\
\hline $\mathrm{BP}$ & GO:0072009 & Nephron epithelium development & $11 / 230$ & $2.11 \times 10^{-7}$ & 11 \\
\hline $\mathrm{CC}$ & GO:0043025 & Neuronal cell body & $20 / 240$ & $1.12 \times 10^{-6}$ & 20 \\
\hline $\mathrm{CC}$ & GO:0044297 & Cell body & $20 / 240$ & $8.82 \times 10^{-6}$ & 20 \\
\hline $\mathrm{CC}$ & GO:0044420 & Extracellular matrix component & $9 / 240$ & $2.67 \times 10^{-5}$ & 9 \\
\hline $\mathrm{CC}$ & GO:0005604 & Basement membrane & $8 / 240$ & $2.80 \times 10^{-5}$ & 8 \\
\hline $\mathrm{CC}$ & GO:0098644 & Complex of collagen trimers & $4 / 240$ & 0.000113 & 4 \\
\hline MF & GO:0034483 & Heparan sulfate sulfotransferase activity & $4 / 228$ & $3.54 \times 10^{-5}$ & 4 \\
\hline KEGG & hsa00534 & $\begin{array}{l}\text { Glycosaminoglycan biosynthesis-heparan } \\
\text { sulfate/heparin }\end{array}$ & $4 / 113$ & 0.000418 & 4 \\
\hline KEGG & hsa04514 & Cell adhesion molecules (CAMs) & $8 / 113$ & 0.001462 & 8 \\
\hline KEGG & hsa04974 & Protein digestion and absorption & $6 / 113$ & 0.00233 & 6 \\
\hline KEGG & hsa04512 & ECM-receptor interaction & $5 / 113$ & 0.007853 & 5 \\
\hline KEGG & hsa00590 & Arachidonic acid metabolism & $4 / 113$ & 0.014923 & 4 \\
\hline
\end{tabular}

BP, biological process; CC, cellular component; GO, Gene Ontology; KEGG, Kyoto Encyclopedia of Genes and Genomes; MF, molecular function.

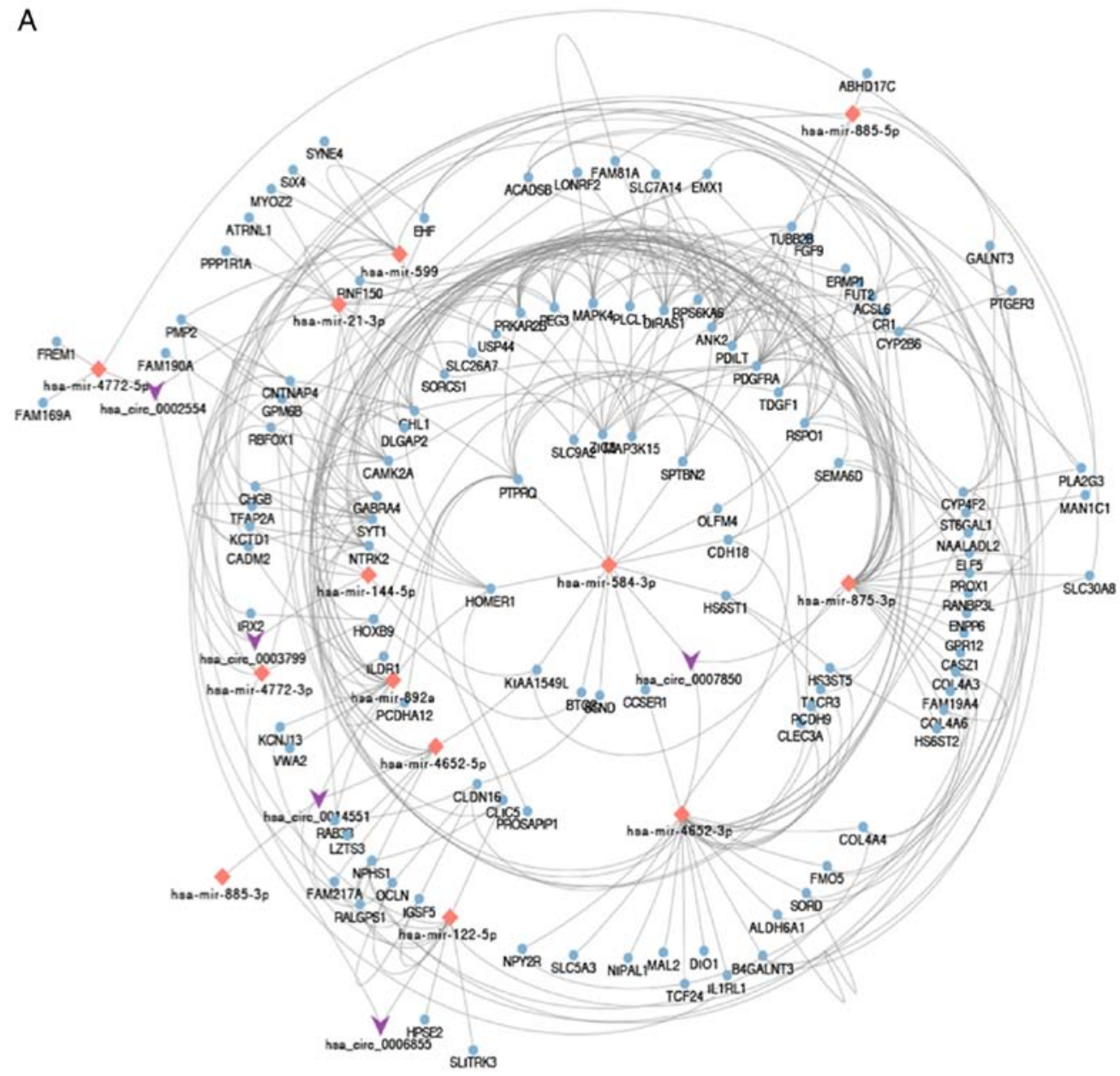

Figure 3. Enrichment analysis of the circRNA-miRNA-downregulated mRNA network. (A) Regulatory network of hub circRNAs. Arrowheads represent circRNAs, diamonds represent miRNAs and circles represent genes. 


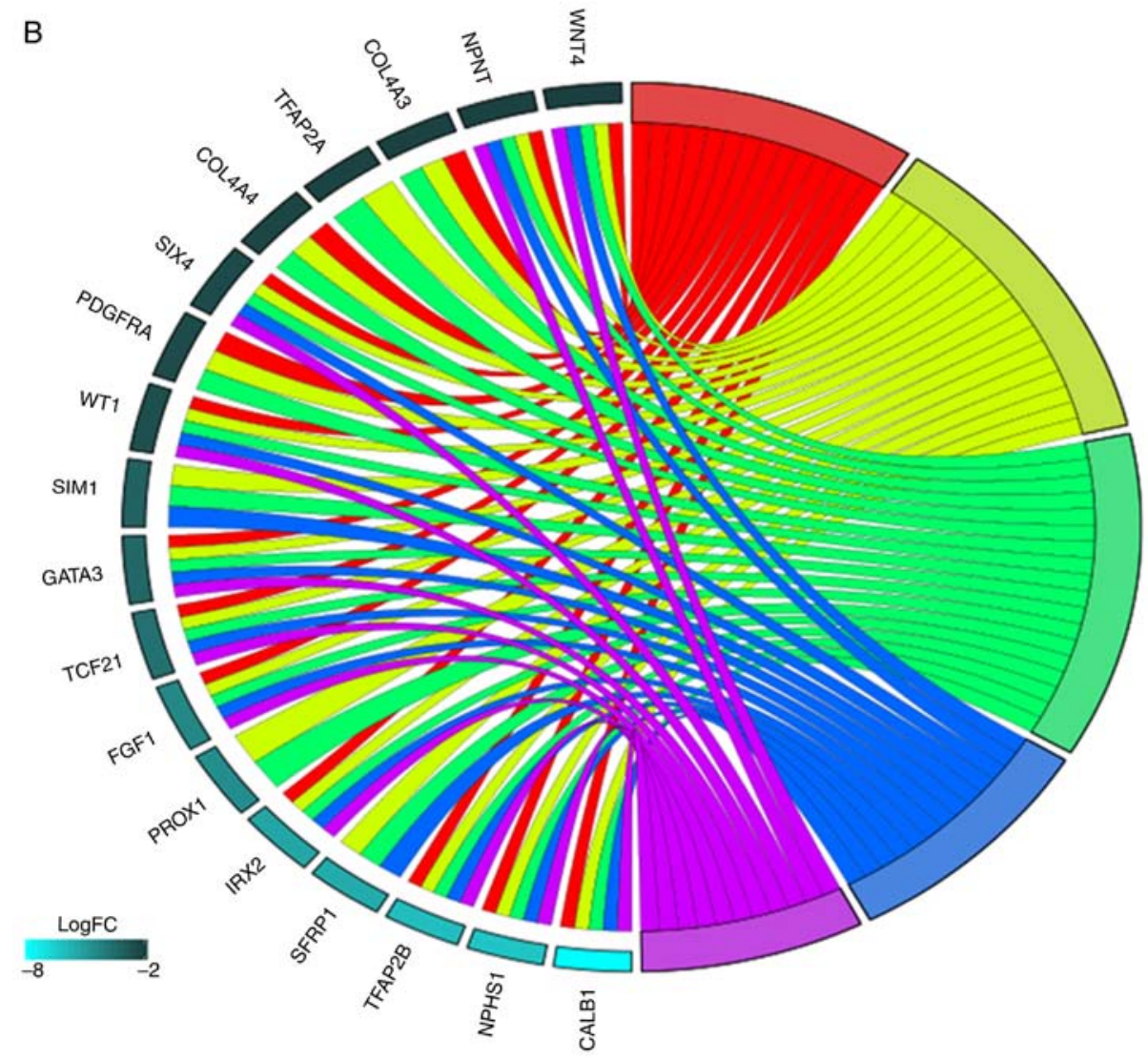
GO terms
Nephron development
$\square$ Kidney development
$\square$ Renal system development
Nephron epithelium development
Kidney epithelium development

C

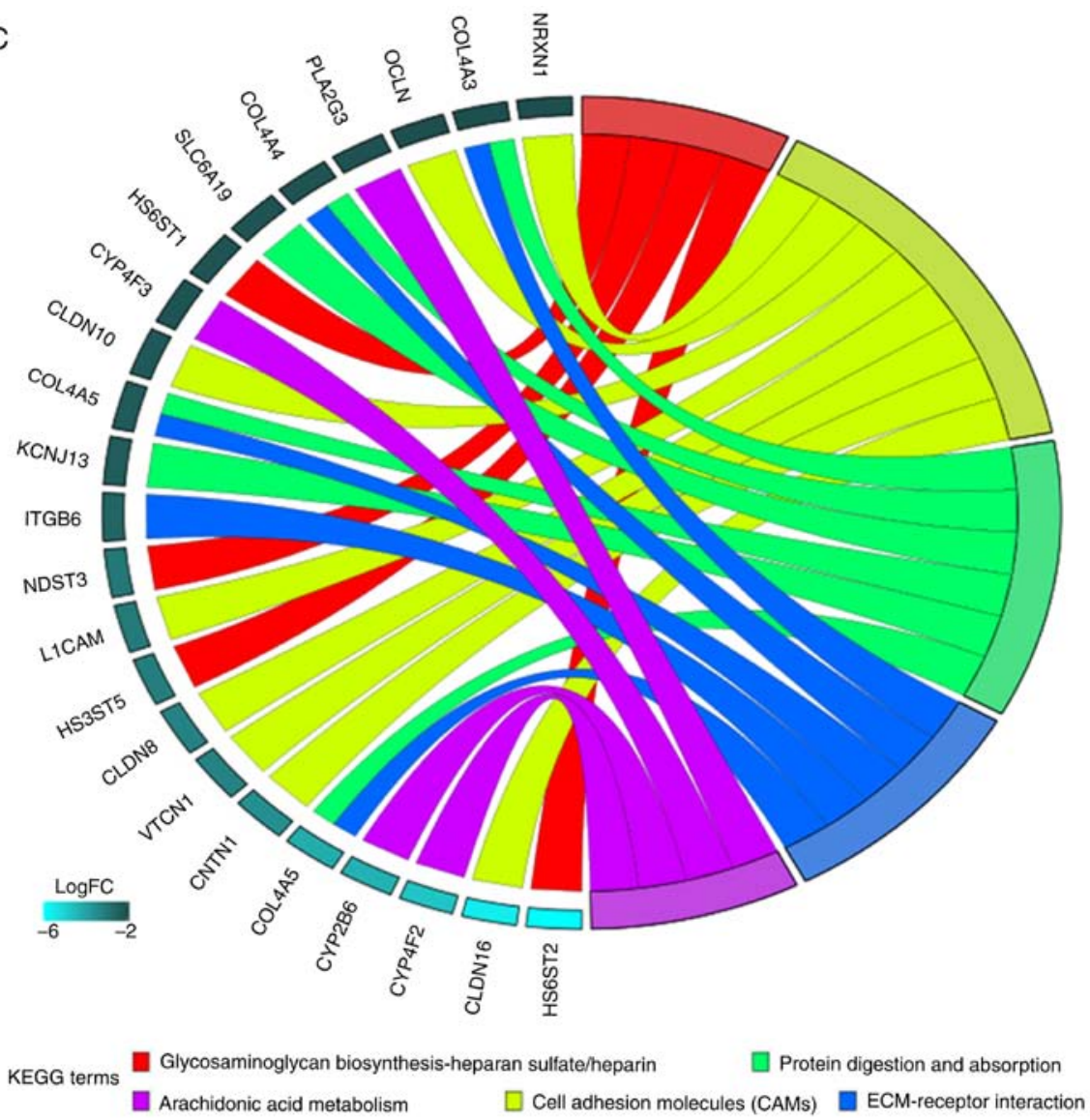

Figure 3. Continued. (B) Top 5 GO terms and (C) top 5 KEGG pathways, as determined by enrichment analysis. circRNA, circular RNA; GO, Gene Ontology; KEGG, Kyoto Encyclopedia of Genes and Genomes; miRNA, microRNA. 
Enrichment analysis was performed on the 244 downregulated genes. As presented in Fig. 3B and Table III, DEGs were enriched in BP, including 'nephron development', 'kidney development' and 'renal system development'. CC analysis suggested that DEGs were associated with 'neuronal cell body', 'cell body' and 'extracellular matrix component'. In addition, these DEGs were significantly enriched in MF, including 'heparan sulfate sulfotransferase activity'. KEGG enrichment pathway analysis revealed DEGs to be involved in 'ECM-receptor interaction', 'glycosaminoglycan biosynthesis-heparan sulfate/heparin' and 'cell adhesion molecules (CAMs)' (Fig. 3C and Table III).

Prognostic value of miRNAs and mRNAs regulated by circRNAs. To identify potential prognostic indicators of CCRCC, the miRNAs and mRNAs regulated by circRNAs were analyzed. Following prognostic analysis, 125 genes and 10 miRNAs were associated with the prognosis of CCRCC. Subsequently, as shown in Fig. 4 and Table IV, the genes and miRNAs were sorted based on P-value, after which the top 10 genes and miRNAs were selected as hub genes [membrane palmitoylated protein 7 (MPP7), aldehyde dehydrogenase 6 family member A1 (ALDH6A1), transcription factor AP-2 $\alpha$ (TFAP2A), collagen type IV $\alpha 4$ chain (COL4A4), nuclear receptor subfamily 3 group $\mathrm{C}$ member 2 (NR3C2), plasminogen (PLG), Holliday junction recognition protein (HJURP), claudin 10 (CLDN10), kinesin family member 18B (KIF18B) and thyroid hormone receptor $\beta$ (THRB)] and hub miRNAs (hsa-miR-21-3p, hsa-miR-155-3p, hsa-miR-144-3p, hsa-miR-142-5p, hsa-miR-875-3p, hsa-miR-885-3p, hsa-miR-3941, hsa-miR-224-3p, hsa-miR-584-3p and hsa-miR-138-1-3p).

\section{Discussion}

ceRNAs are involved in a complex regulatory network associated with the transcriptome; the present findings may improve understanding of the regulatory mechanism underlying gene expression. The present findings, along with other studies $(9,11,18)$, suggest the importance of ceRNAs in the carcinogenesis of various types of cancer. The present study analyzed GEO and TCGA datasets to identify DECs, DEMs and DEGs in CCRCC. In addition, ceRNA regulatory networks in CCRCC were constructed using these circRNAs, miRNAs and genes. Additionally, the prognostic value of miRNAs and genes regulated by circRNAs was determined to identify indicators that may predict the prognosis of CCRCC.

By analyzing the data from a circRNA expression microarray of CCRCC (GSE100186), which contained four CCRCC samples and four normal samples, 324 downregulated and 218 upregulated circRNAs were reported in the cancer group. The mRNA and miRNA expression profiles of TCGA CCRCC dataset were used to identify DEGs and DEMs between cancer and normal samples. Using the CSCD, TargetScan and miRDB, a circRNA-miRNA-mRNA regulatory network was constructed in CCRCC based on the ceRNA theory.

The results of enrichment analysis of the 85 upregulated genes in the circRNA-miRNA-upregulated mRNA network suggested associated BP terms, including 'flavonoid metabolic process', 'cellular glucuronidation', 'uronic acid metabolic process', 'glucuronate metabolic process' and 'response to
Table IV. Prognostic analysis of hub genes and miRNAs.

\begin{tabular}{llll}
\hline Gene & HR & $95 \%$ CI & P-value \\
\hline MPP7 & 0.397 & $0.293-0.538$ & $1.42 \times 10^{-9}$ \\
ALDH6A1 & 0.388 & $0.287-0.525$ & $1.70 \times 10^{-9}$ \\
TFAP2A & 2.649 & $1.961-3.578$ & $3.63 \times 10^{-9}$ \\
COL4A4 & 0.398 & $0.295-0.538$ & $7.88 \times 10^{-9}$ \\
NR3C2 & 0.396 & $0.293-0.536$ & $1.79 \times 10^{-8}$ \\
PLG & 0.411 & $0.304-0.556$ & $3.15 \times 10^{-8}$ \\
HJURP & 2.455 & $1.817-3.319$ & $5.84 \times 10^{-8}$ \\
CLDN10 & 0.432 & $0.319-0.584$ & $8.22 \times 10^{-8}$ \\
KIF18B & 2.415 & $1.787-3.265$ & $9.02 \times 10^{-8}$ \\
THRB & 0.441 & $0.326-0.596$ & $9.42 \times 10^{-8}$ \\
hsa-mir-21-3p & 2.745 & $2.029-3.712$ & $7.12 \times 10^{-11}$ \\
hsa-mir-155-3p & 1.702 & $1.262-2.295$ & $5.72 \times 10^{-4}$ \\
hsa-mir-144-3p & 0.625 & $0.463-0.843$ & 0.002 \\
hsa-mir-142-5p & 1.587 & $1.176-2.14$ & 0.003 \\
hsa-mir-875-3p & 0.626 & $0.462-0.849$ & 0.004 \\
hsa-mir-885-3p & 0.649 & $0.481-0.876$ & 0.005 \\
hsa-mir-3941 & 0.71 & $0.526-0.958$ & 0.025 \\
hsa-mir-224-3p & 1.371 & $1.017-1.849$ & 0.039 \\
hsa-mir-584-3p & 0.734 & $0.544-0.989$ & 0.045 \\
hsa-mir-138-1-3p & 1.35 & $1.001-1.822$ & 0.048 \\
\hline & & & \\
\hline
\end{tabular}

miR/miRNA, microRNA.

xenobiotic stimulus'. According to the results, CCRCC was associated with various metabolic processes, including flavonoid, uronic acid and glucuronate metabolism. The exact role of the various metabolic pathways in the initiation, progression and treatment of CCRCC requires further investigation. KEGG enrichment analysis indicated the importance of 'ascorbate and aldarate metabolism', 'pentose and glucuronate interconversions', 'steroid hormone biosynthesis' and 'retinol metabolism'. Steroid hormones serve a critical role in the regulation of metabolism, inflammation, immune functions, salt and water balance, the development of sexual characteristics, and the ability to withstand illness and injury (19). It has previously been reported that glucocorticoids inhibit the development of renal cancer by increasing the levels of $\mathrm{Na}$ and the expression of K-ATPase $\beta-1$ subunit, which suggests the possible benefits of glucocorticoids as a supplementary treatment in RCC management (20). In addition, aldosterone mediates the metastatic spread of renal cancer via its $G$ protein-coupled estrogen receptor (GPER); therefore, GPER inhibitors may be considered promising therapeutic agents for inhibiting metastatic spread (21). Further study into the specific molecular mechanisms underlying the effects of steroid hormones on CCRCC development is required.

In the present study, enrichment analysis was performed on the 244 downregulated genes associated with the circRNA-miRNA-downregulated mRNA network. The results indicated critical BP terms, including 'nephron development', 'kidney development' and 'renal system development'. In addition, MF analysis revealed 'heparan sulfate sulfotransferase activity' was enriched in this network, whereas KEGG 

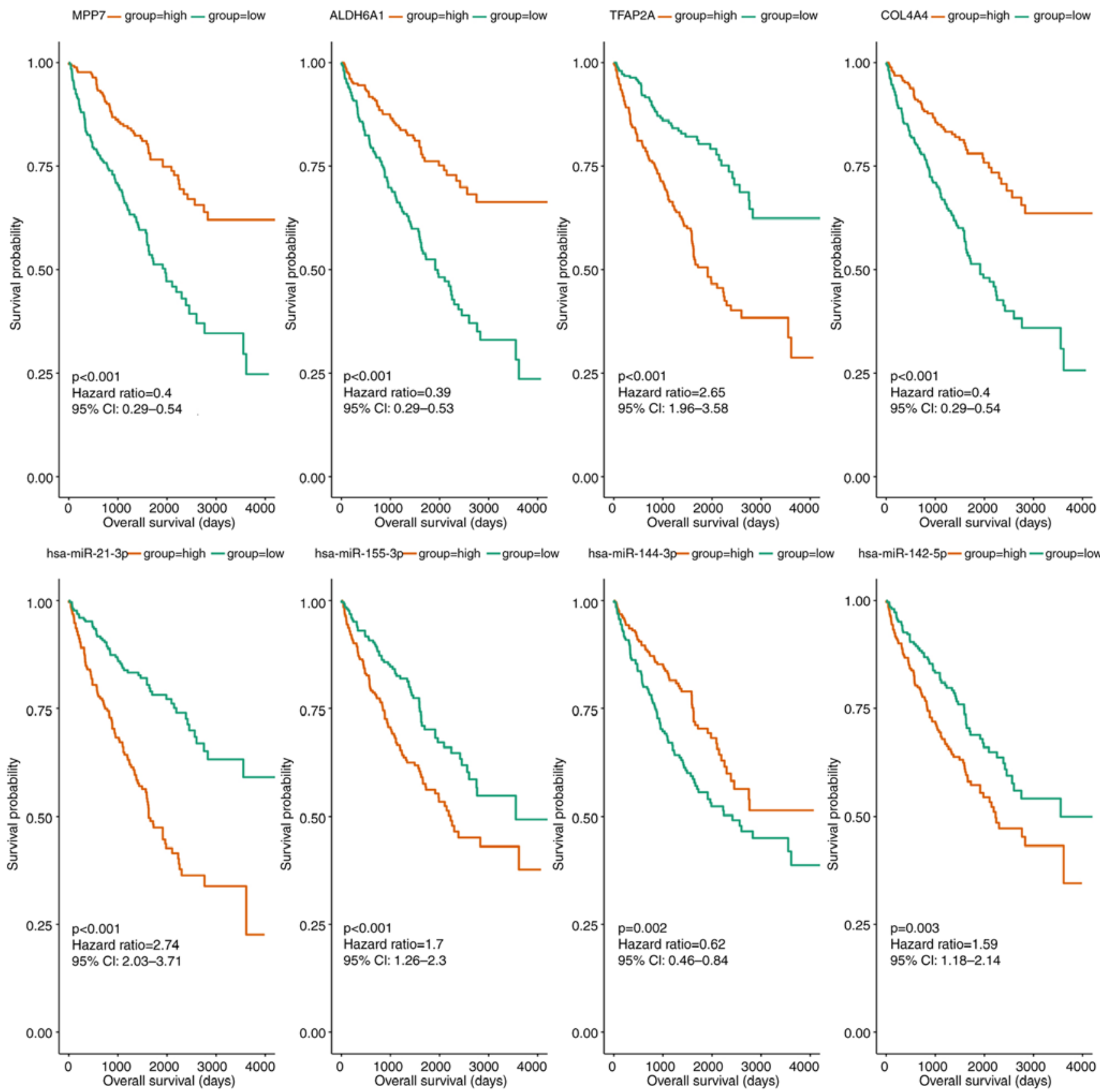

Figure 4. Prognostic analysis of the top four genes and miRNAs in relation to clear cell renal cell carcinoma survival. ALDH6A1, aldehyde dehydrogenase 6 family member A1; COL4A4, collagen type IV $\alpha 4$ chain; miR/miRNA, microRNA; MPP7, membrane palmitoylated protein 7; TFAP2A, transcription factor AP-2 $\alpha$.

pathway enrichment analysis suggested the importance of 'ECM-receptor interaction', 'glycosaminoglycan biosynthesis-heparan sulfate/heparin' and 'cell adhesion molecules (CAMs)', 'ECM-receptor interaction' and 'arachidonic acid metabolism'. CAMs are a subset of proteins that maintain cellular polarity and inhibit tumor growth (22). Cell adhesion molecule 4, which is one of the immunoglobulin-superfamily CAM proteins, has been proposed to be involved in suppressing tumor invasion and formation in CCRCC and nude mice (23). In addition, dysregulated methylation and suppression of the tumor inhibitor cell adhesion molecule 2 (CADM2) have been linked to human renal cell carcinogenesis; therefore, CADM2 could be a possible therapeutic target (24). These findings suggested that analysis of biological terms may provide novel insight into the complex mechanisms underlying the development and progression of CCRCC.

In order to identify prognostic indicators of CCRCC, the miRNAs and genes regulated by circRNAs were investigated. According to the analysis, the top 10 genes and miRNAs were selected as hub genes (MPP7, ALDH6A1, TFAP2A, COL4A4, NR3C2, PLG, HJURP, CLDN10, KIF18B and THRB) and hub miRNAs (miR-21-3p, miR-155-3p, miR-144-3p, miR-142-5p, miR-875-3p, miR-885-3p, miR-3941, miR-224-3p, miR-584-3p and miR-138-1-3p). The tumor suppressor gene TFAP2A has been reported to be hypermethylated and markedly downregulated in RCC. Therefore, analysis of TFAP2A methylation in cells obtained from urine or blood samples may be valuable in early diagnosis (25). The suppressive role of NR3C2 has 
been reported in various types of cancer; low NR3C2 expression levels are correlated with aggressive characteristics and poorer survival in non-distant metastatic CCRCC (26). In a study investigating the role of gene copy number variation in relation to the clinical parameters of metastatic CCRCC, the loss of PLG was associated with advanced tumor stage and Fuhrman grade (27). As for the hub miRNAs, miR-21 has been widely studied in renal cancer for its regulatory roles in cellular proliferation and metastasis (28-30). In addition, the hub miRNA, miR-155, was determined to regulate the growth and invasion of CCRCC cells by interacting with E2F transcription factor 2 (31). miR-155 has also been suggested to modulate the proliferation, invasion and apoptosis of renal carcinoma cells by altering the glycogen synthase kinase- $3 \beta / \beta$-catenin pathway (32). Furthermore, miR-144-3p could promote cell proliferation and migration in CCRCC by downregulating AT-rich interactive domain-containing protein 1A (33), which was also regarded as a possible novel plasma biomarker for the diagnosis of CCRCC (34). This study identified numerous ceRNAs that may serve a critical role in the development of CCRCC; however, the specific mechanism as to how these ceRNAs function requires further investigation.

In the present study, a series of circRNAs, miRNAs and genes, which may be implicated in CCRCC, were identified. In addition, the ceRNA regulatory network of circRNAs-miRNAs-genes was constructed, and could serve as a wide-scale profile of the complex regulation underlying the development of CCRCC. These findings may not only provide insight into the etiology of CCRCC, but could aid developments into the treatment of this disease.

\section{Acknowledgements}

Not applicable.

\section{Funding}

No funding was received.

\section{Availability of data and materials}

The dataset generated and/or analyzed during the current study are available in TCGA (https://cancergenome.nih.gov/) and GEO (https://www.ncbi.nlm.nih.gov/gds/).

\section{Authors' contributions}

CM, JQ and XLW analysed the data, CM, JPZ and DJW wrote the manuscript, and XNL designed the study and wrote and revised the article manuscript. JPZ and DJW identified the databases and reviewed the data analysis. All authors read and approved the final manuscript.

\section{Ethics approval and consent to participate}

Not applicable.

\section{Patient consent for publication}

Not applicable.

\section{Competing interests}

The authors declare that they have no competing interests.

\section{References}

1. Schmidt LS and Linehan WM: Genetic predisposition to kidney cancer. Semin Oncol 43: 566-574, 2016.

2. Morris MR and Latif F: The epigenetic landscape of renal cancer. Nat Rev Nephrol 13: 47-60, 2017.

3. Moch H, Srigley J, Delahunt B, Montironi R, Egevad L and Tan PH: Biomarkers in renal cancer. Virchows Arch 464: 359-365, 2014.

4. Dabestani S, Marconi L and Bex A: Metastasis therapies for renal cancer. Curr Opin Urol 26: 566-572, 2016.

5. Lu CH, Ou YC, Huang LH, Weng WC, Chang YK, Chen HL, Hsu CY and Tung MC: Early dutasteride monotherapy in patients with elevated serum prostate-specific antigen levels following robot-assisted radical prostatectomy. Front Oncol 9: $691,2019$.

6. Corgna E, Betti M, Gatta G, Roila F and De Mulder PH: Renal cancer. Crit Rev Oncol Hematol 64: 247-262, 2007.

7. Zhao ZJ and Shen J: Circular RNA participates in the carcinogenesis and the malignant behavior of cancer. RNA Biol 14: 514-521, 2017.

8. Salzman J: Circular RNA expression: Its potential regulation and function. Trends Genet 32: 309-316, 2016.

9. Meng X, Li X, Zhang P, Wang J, Zhou Y and Chen M: Circular RNA: An emerging key player in RNA world. Brief Bioinform 18: 547-557, 2017.

10. Zhang HD, Jiang LH, Sun DW, Hou JC and Ji ZL: CircRNA: A novel type of biomarker for cancer. Breast Cancer 25: 1-7, 2018.

11. Ebbesen KK, Hansen TB and Kjems J: Insights into circular RNA biology. RNA Biol 14: 1035-1045, 2017.

12. Wang K, Sun Y, Tao W, Fei X and Chang C: Androgen receptor (AR) promotes clear cell renal cell carcinoma (ccRCC) migration and invasion via altering the circHIAT1/miR-195-5p/29a-3p/29c-3p/CDC42 signals. Cancer Lett 394: 1-12, 2017.

13. Wang G, Xue W, Jian W, Liu P, Wang Z, Wang C, Li H, Yu Y, Zhang D and Zhang C: The effect of Hsa_circ_0001451 in clear cell renal cell carcinoma cells and its relationship with clinicopathological features. J Cancer 9: 3269-3277, 2018.

14. Xiong Y, Zhang J and Song C: CircRNA ZNF609 functions as a competitive endogenous RNA to regulate FOXP4 expression by sponging miR-138-5p in renal carcinoma. J Cell Physiol 234: 10646-10654, 2019.

15. Xia S, Feng J, Chen K, Ma Y, Gong J, Cai F, Jin Y, Gao Y, Xia L, Chang H, et al: CSCD: A database for cancer-specific circular RNAs. Nucleic Acids Res 46: D925-D929, 2018.

16. The Gene Ontology (GO) project in 2006. Nucleic Acids Res 34: D322-D326, 2006.

17. Kanehisa M and Goto S: KEGG: Kyoto encyclopedia of genes and genomes. Nucleic Acids Res 28: 27-30, 2000.

18. Sanchez-Mejias A and Tay Y: Competing endogenous RNA networks: Tying the essential knots for cancer biology and therapeutics. J Hematol Oncol 8: 30, 2015.

19. D'Uva G and Lauriola M: Towards the emerging crosstalk: ERBB family and steroid hormones. Semin Cell Dev Biol 50: 143-152, 2016.

20. Huynh TP, Barwe SP, Lee SJ, McSpadden R, Franco OE, Hayward SW, Damoiseaux R, Grubbs SS, Petrelli NJ and Rajasekaran AK: Glucocorticoids suppress renal cell carcinoma progression by enhancing $\mathrm{Na}, \mathrm{K}-\mathrm{ATP}$ ase beta-1 subunit expression. PLoS One 10: e0122442, 2015.

21. Feldman RD, Ding Q, Hussain Y, Limbird LE, Pickering JG and Gros R: Aldosterone mediates metastatic spread of renal cancer via the $\mathrm{G}$ protein-coupled estrogen receptor (GPER). FASEB J 30: 2086-2096, 2016.

22. Zhong X, Drgonova J, Li CY and Uhl GR: Human cell adhesion molecules: Annotated functional subtypes and overrepresentation of addiction-associated genes. Ann N Y Acad Sci 1349: 83-95, 2015.

23. Nagata M, Sakurai-Yageta M, Yamada D, Goto A, Ito A, Fukuhara H, Kume H, Morikawa T, Fukayama M, Homma Y and Murakami Y: Aberrations of a cell adhesion molecule CADM4 in renal clear cell carcinoma. Int J Cancer 130: 1329-1337, 2012. 
24. He W, Li X, Xu S, Ai J, Gong Y, Gregg JL, Guan R, Qiu W, Xin D, Gingrich JR, et al: Aberrant methylation and loss of CADM2 tumor suppressor expression is associated with human renal cell carcinoma tumor progression. Biochem Biophys Res Commun 435: 526-532, 2013.

25. Dalgin GS, Drever M, Williams T, King T, DeLisi C and Liou LS Identification of novel epigenetic markers for clear cell renal cell carcinoma. J Urol 180: 1126-1130, 2008.

26. Zhao Z, Zhang M, Duan X, Deng T, Qiu H and Zeng G: Low NR3C2 levels correlate with aggressive features and poor prognosis in non-distant metastatic clear-cell renal cell carcinoma. J Cell Physiol 233: 6825-6838, 2018.

27. Nouhaud FX, Blanchard F, Sesboue R, Flaman JM, Sabourin JC, Pfister C and Di Fiore F: Clinical relevance of gene copy number variation in metastatic clear cell renal cell carcinoma. Clin Genitourin Cancer 16: e795-e805, 2018.

28. An F, Liu Y and Hu Y: miR-21 inhibition of LATS1 promotes proliferation and metastasis of renal cancer cells and tumor stem cell phenotype. Oncol Lett 14: 4684-4688, 2017.

29. Bera A, Ghosh-Choudhury N, Dey N, Das F, Kasinath BS Abboud HE and Choudhury GG: NFkB-mediated cyclin D1 expression by microRNA-21 influences renal cancer cell proliferation. Cell Signal 25: 2575-2586, 2013.

30. Yuan H, Xin S, Huang Y, Bao Y, Jiang H, Zhou L, Ren X, Li L, Wang Q and Zhang J: Downregulation of PDCD4 by miR-21 suppresses tumor transformation and proliferation in a nude mouse renal cancer model. Oncol Lett 14: 3371-3378, 2017.

31. Gao Y, Ma X, Yao Y, Li H, Fan Y, Zhang Y, Zhao C, Wang L, Ma M, Lei $Z$ and Zhang X: miR-155 regulates the proliferation and invasion of clear cell renal cell carcinoma cells by targeting E2F2. Oncotarget 7: 20324-20337, 2016.

32. Wei RJ, Zhang CH and Yang WZ: MiR-155 affects renal carcinoma cell proliferation, invasion and apoptosis through regulating GSK-3 $\beta / \beta$-catenin signaling pathway. Eur Rev Med Pharmacol Sci 21: 5034-5041, 2017.
33. Xiao W, Lou N, Ruan H, Bao L, Xiong Z, Yuan C, Tong J, Xu G, Zhou Y, Qu Y, et al: Mir-144-3p promotes cell proliferation, metastasis, sunitinib resistance in clear cell renal cell carcinoma by downregulating ARID1A. Cell Physiol Biochem 43: 2420-2433, 2017

34. Lou N, Ruan AM, Qiu B, Bao L, Xu YC, Zhao Y, Sun RL, Zhang ST, Xu GH, Ruan HL, et al: miR-144-3p as a novel plasma diagnostic biomarker for clear cell renal cell carcinoma. Urol Oncol 35: 36.e7-36.e14, 2017.

35. Salzman J, Chen RE, Olsen MN, Wang PL and Brown PO: Cell-type specific features of circular RNA expression. PLoS Genet 9: e1003777, 2013.

36. Jeck WR, Sorrentino JA, Wang K, Slevin MK, Burd CE, Liu J, Marzluff WF and Sharpless NE: Circular RNAs are abundant, conserved, and associated with ALU repeats. RNA 19: 141-157, 2013.

37. Rybak-Wolf A, Stottmeister C, Glažar P, Jens M, Pino N, Giusti S, Hanan M, Behm M, Bartok O, Ashwal-Fluss R, et al: Circular RNAs in the mammalian brain are highly abundant, conserved, and dynamically expressed. Mol Cell 58: 870-885, 2015.

38. Memczak S, Jens M, Elefsinioti A, Torti F, Krueger J, Rybak A, Maier L, Mackowiak SD, Gregersen LH, Munschauer M, et al: Circular RNAs are a large class of animal RNAs with regulatory potency. Nature 495: 333-338, 2013.

39. Zhang Y, Zhang XO, Chen T, Xiang JF, Yin QF, Xing YH, Zhu S, Yang L and Chen LL: Circular intronic long noncoding RNAs. Mol Cell 51: 792-806, 2013

This work is licensed under a Creative Commons Attribution-NonCommercial-NoDerivatives 4.0 International (CC BY-NC-ND 4.0) License. 